\title{
Recent Micro-CT Scanner Developments at UGCT
}

\author{
Manuel Dierick ${ }^{1,2}$, Denis Van Loo ${ }^{1,2}$, Bert Masschaele ${ }^{1,2}$, Matthieu Boone ${ }^{1}$, Luc Van \\ Hoorebeke $^{1}$ and Veerle Cnudde ${ }^{3}$ \\ ${ }^{1}$ UGCT-Department of Physics and Astronomy - SHE, Faculty of Sciences, Ghent University, \\ Proeftuinstraat 86, 9000 Ghent, Belgium [email: manuel.dierick@UGent.be] \\ ${ }^{2}$ XRE, X-Ray Engineering bvba, De Pintelaan 111, 9000 Ghent, Belgium [email: info@XRE.be] \\ ${ }^{3}$ UGCT-Department of Geology and Soil Science - SHE, Faculty of Sciences, Ghent University, \\ Krijgslaan 281, S8, 9000 Ghent, Belgium [email: Veerle.Cnudde@UGent.be]
}

Keywords: X-ray micro-tomography, Micro-CT scanners, tomography acquisition, image processing

\begin{abstract}
UGCT is a user facility for multidisciplinary micro-CT research. The scanners at UGCT are custom designed and built by the Radiation Physics research group (UGent). This paper describes the two latest scanners that were developed in collaboration with XRE: HECTOR, a high energy micro-CT scanner, and EMCT, a gantry based micro-CT scanner with variable magnification. HECTOR is a 240 $\mathrm{kV} 280 \mathrm{~W}$ system with a nominal resolution of 4 micrometer. A $40 \times 40 \mathrm{~cm}^{2}$ flat panel detector which can be tiled results in a field of view of $80 \times 80 \mathrm{~cm}^{2}$. The sample positioning stage can carry samples up to $80 \mathrm{~kg}$.

The second scanner, EMCT, is a gantry based micro-focus system with a variable magnification. Unlike medical or small-animal scanners, the rotation axis is mounted vertically. It was designed to allow scans of objects that are hard to rotate in a standard micro-CT scanner, such as samples that are wired to peripheral equipment or samples in a fluid environment. The source is a $130 \mathrm{kV} 15 \mathrm{~W}$ microfocus source (nominal resolution $5 \mu \mathrm{m}$ ) with integrated high-voltage supply. The standard detector is a $15 \times 15 \mathrm{~cm}^{2}$ flat panel detector resulting in a maximal field-of-view of $12 \mathrm{~cm}$ diameter.

In addition a high-speed detector is available which can be installed on both scanners for micro-CT of dynamic processes. To further extend the effective X-ray energy at HECTOR a high sensitivity line detector will be installed in the near future.
\end{abstract}

\section{INTRODUCTION}

UGCT is a user facility for multidisciplinary micro-CT research [1]. Within UGCT, the Radiation Physics research group is responsible for the technological developments of micro-tomography itself as a tool for multidisciplinary research. This includes the design and construction of the actual scanners, and the development of control- and acquisition software [2], reconstruction software (Octopus) [3] and 3D analysis software (Morpho+) [4].

The previously available scanners at UGCT were primarily designed for high-resolution CT. As a result they are limited to $160 \mathrm{kV}$, which puts a limit on the size and composition of the samples in order to have sufficient transmission to allow tomographic reconstruction. Additionally, they were not designed for high-speed scanning. To overcome these limitations, a more powerful high energy system called HECTOR has been developed, which is nevertheless capable of achieving high resolutions. Another limitation of the existing scanners is the fact that the sample is rotated in between a fixed sourcedetector setup. In several applications it is hard to rotate the sample, for example because of wiring or tubing that is attached to the sample. For such cases the gantry based system EMCT was designed which rotates the whole tube-detector assembly around a stationary sample. The challenge was to achieve a resolution of 5 micrometer while keeping the flexibility high by making the source-detector distance variable, unlike most other gantry based CT scanners such as medical or small animal scanners which typically have a magnification which is fixed or only variable over a small range.

\section{SPECIFICATIONS AND FEATURES OF HECTOR}

For the source, a $240 \mathrm{kV}$ open type directional source was chosen with a spot-size small enough to achieve $4 \mu \mathrm{m}$ resolution and a maximal power of $280 \mathrm{~W}$. The rotation stage has a mechanical bearing 
with a total positional error of less than $2 \mu \mathrm{m}$ but is nonetheless capable of handling axial loads up to $80 \mathrm{~kg}$. For routine scanning, a large $40 \times 40 \mathrm{~cm}$ flat panel with $(200 \mu \mathrm{m})^{2}$ pixels covered with Csl scintillator was installed. The detector mount has an additional flange for either a high speed detector or a line detector which will be installed in the near future. The detector can be moved along the translational and vertical direction to extend the field-of-view to $80 \times 80 \mathrm{~cm}^{2}$. This was mechanically aligned and calibrated to sub-pixel precision in such a way that the images can simply be assembled without interpolation or registration. The detector stage and the sample stage can move independently along the magnification direction. The source-detector-distance ranges from $30 \mathrm{~cm}$ to $200 \mathrm{~cm}$ while the source-object-distance can range from $3 \mathrm{~mm}$ to $175 \mathrm{~cm}$, covering a magnification range of almost 1 to 600 . HECTOR is constructed in a large concrete bunker with plenty of room to install additional hardware such as climate conditioners or load cells [5].

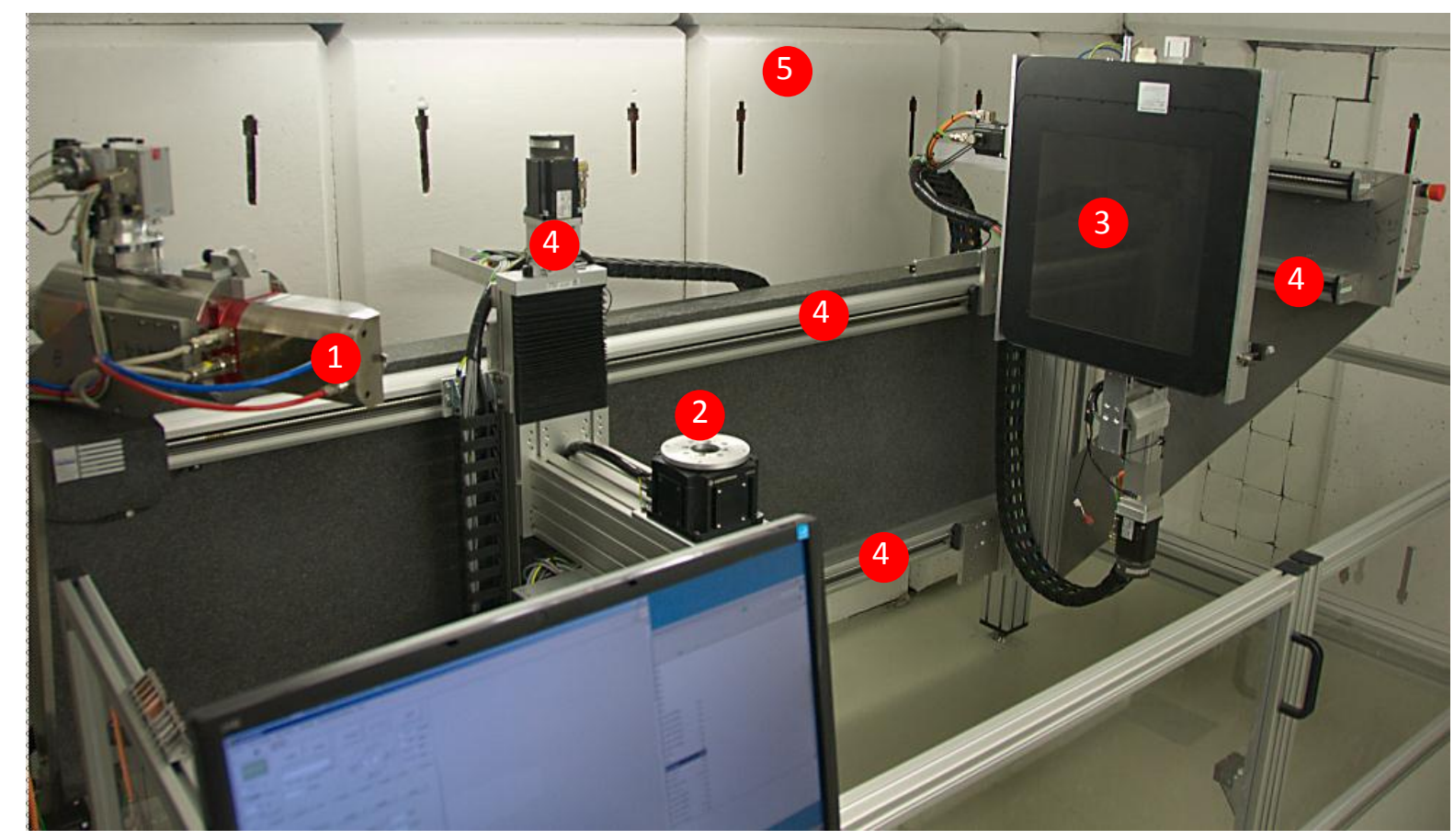

Figure 1: The HECTOR scanner showing the source (1), rotation stage (2), detector pannel (3), positioning stages (4) inside the concrete bunker room (5)

The $280 \mathrm{~W}$ of maximum available power allows for strong filtration of the beam while still leaving sufficient flux. This results in significantly higher energies than at the other UGCT scanners. A line detector will be installed in the near future which will even further increase the detected average energy because of a significantly thicker scintillator which increases the detection efficiency at higher energies. This extends the range of applications to large tree logs, geological or concrete drill cores, bronze artwork, metal parts etc.

The high power beam also makes it possible to perform high speed CT scanning of dynamic processes. This allows monitoring of processes like foam collapse, water ingress etc. in 3D.

Finally, HECTOR was designed to provide a high degree of flexibility for CT research. This includes support for helical scanning using a high-precision vertical stage with $95 \mathrm{~cm}$ travel. At the rotation stage there is access to a power supply, a UTP connection and several $1 / O$ lines.

The large source-detector-distance is not only useful for scanning large samples but also for phase contrast imaging experiments.

Finally, the detector frame is motorised so it can be rotated $+/-30$ degrees around a vertical axis. This will be used for special acquisition schemes such as limited angle CT, laminography or tomosynthesis methods. 


\section{SPECIFICATIONS AND FEATURES OF EMCT}

The source on the EMCT scanner is a $130 \mathrm{kV}$ closed type directional source with a minimal spot-size of $5 \mu \mathrm{m}$ and a maximal power of $39 \mathrm{~W}$. The X-ray detector, chosen for the EMCT, is a CMOS type with a $400 \mu \mathrm{m}$ Csl scintillator. It has $1358 \times 1356$ pixels of $(0.1 \mathrm{~mm})^{2}$, which leads to a sensitive area of $13.5 \times 13.5 \mathrm{~cm}^{2}$. The source and detector are mounted on a linear motorised stage. Since the samples are stationary with regard to the outside world, this stage can be used to change the magnification of the X-ray system. This magnification stage with detector and source is mounted on a large goniometer (rotation stage). The goniometer has a positional accuracy of less than $5 \mu \mathrm{m}$ and a bore hole of 190 $\mathrm{mm}$ diameter through which the samples can be positioned into the beam. The entire system is built on a granite base to reach the required geometrical precision and stability during the CT scans. The system controller is a compact industrial PC which is also mounted on top of the rotation stage. Communication, interlocks and power are transmitted over slip ring contacts which are embedded in the granite base. This allows for continuous rotation of the scanner around the sample. The detectorsource distance can be changed manually from $150 \mathrm{~mm}$ up to $400 \mathrm{~mm}$.
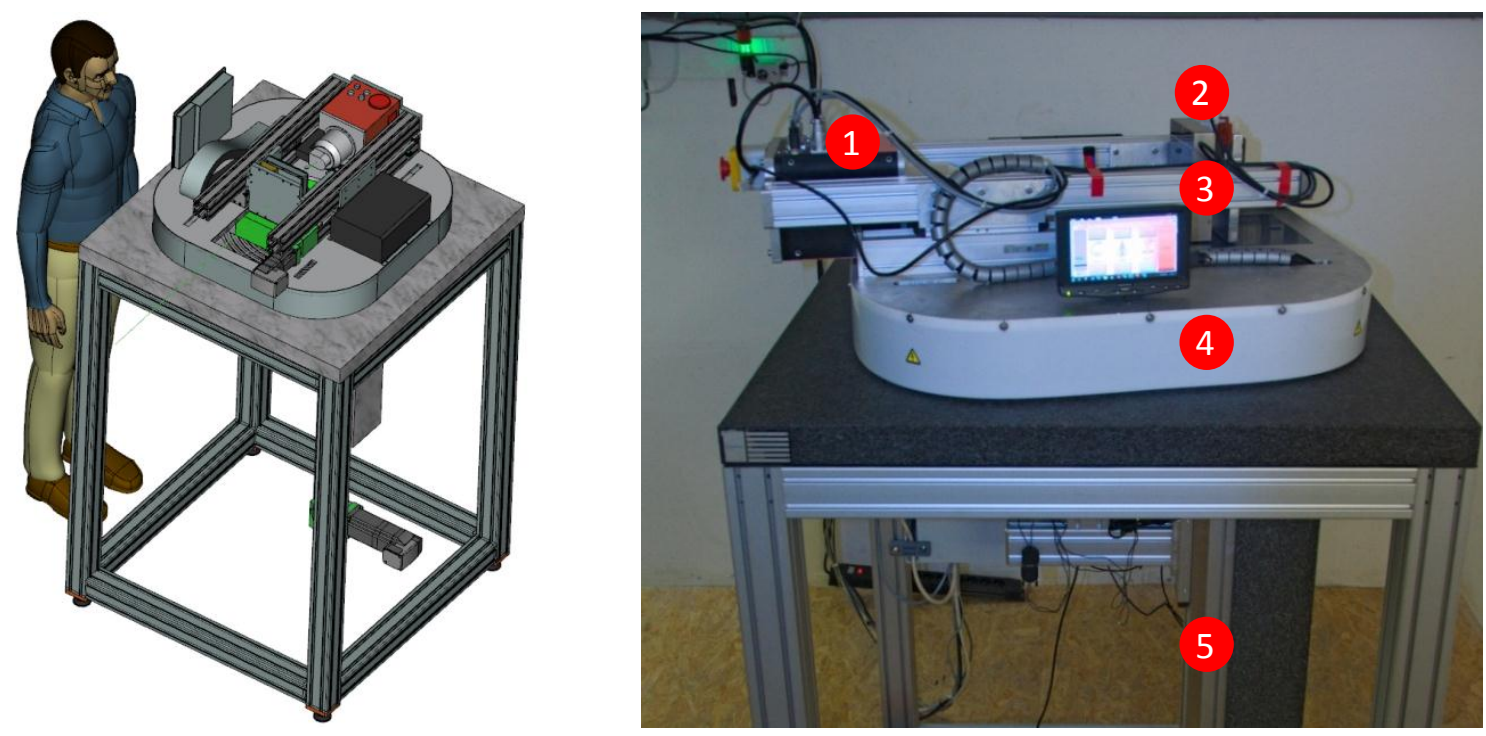

Figure 3: The EMCT scanner CAD design (Left) and actual appearance (Right) showing the $X$-ray source (1), detector (2) on the magnification stage (3) in turn mounted on a rotating gantry (4). The vertical stage (5) is fixed to the frame and allows sample positioning and helical scans.

The flat panel detector can be operated in different modes, each with its own maximum frame rate. In panoramic mode, only a central window of 120 lines is read-out with a frame rate of 300 frames per second. In panoramic mode it is possible to perform high speed CT scans, with a sufficiently small angular interval between successive projections, in a matter of seconds. The EMCT scanner has a vertical stage with $80 \mathrm{~cm}$ travel. This allows for helical scanning of long samples, e.g. drill cores. The samples can be centered on the rotation axis of the CT scanner by means of a piezo XY table which is mounted on the vertical stage.

One of the reasons to mount the gantry horizontally, contrary to medical and small animal scanners, was that it is easier to obtain the high mechanical accuracy required to reach $5 \mu \mathrm{m}$ resolution. Another reason was that, in this way, samples can be supported from below rather than from the side, and therefore the support stays out of the field of view. It also provides easier access to the sample area, especially during experiments involving fluids and/or gravity. Figure 4 shows the 3D rendering of 3 stages during a compression test on aluminum foam. The foam was placed in the centre of the scanner prototype, supported at the bottom which stayed in place, and then step-wise loaded from the top. This way, the size and shape of the cells and the supporting struts can be investigated as function of the loading. 


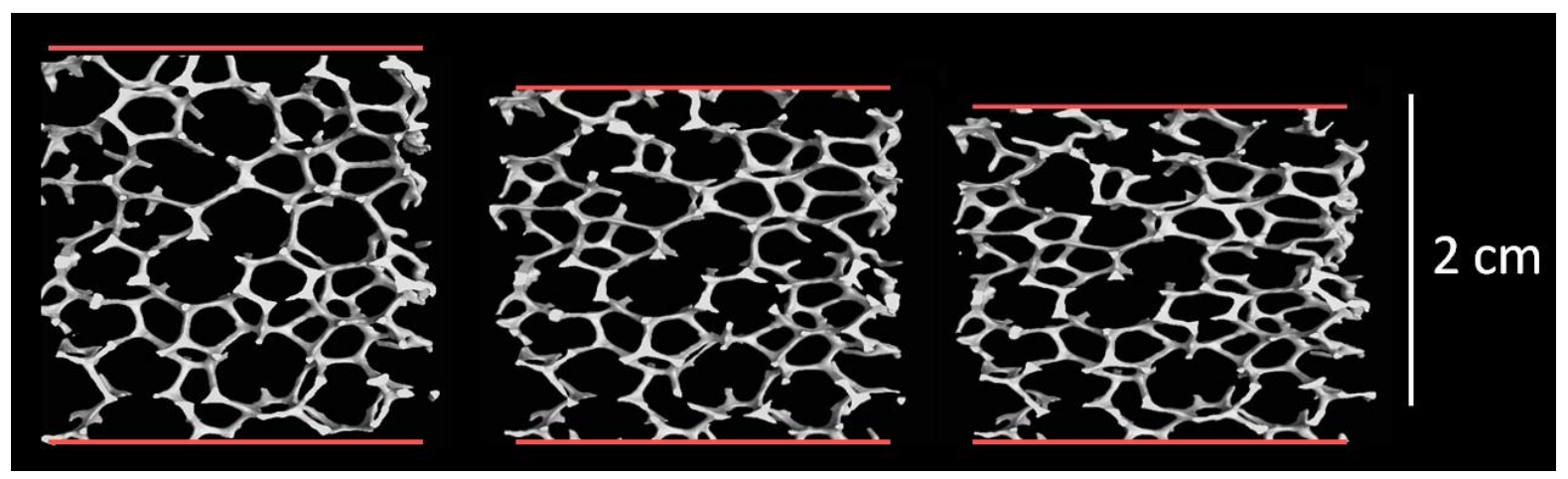

Figure 4: $3 D$ renderings of different stages of compression of aluminum foam.

\section{CONCLUSIONS}

Two new scanners have been developed for use at UGCT that extend the possible range of micro-CT applications in different areas. The higher acceleration voltage and power of HECTOR together with the higher sensitivity of the used detectors have increased the range towards larger and more absorbing samples and opened up the possibility for 4D scanning of dynamic phenomena. The gantry based EMCT scanner allows scanning of objects that can not be rotated in a standard micro-CT scanner. This allows for scanning of samples in a fluid environment, samples inside a climate chamber or a load cell, or samples that are connected to the outside world with wiring, tubing etc.

\section{ACKNOWLEDGEMENTS}

The authors acknowledge the financial support of the Flemish community through the Strategic Initiative Materials in Flanders (SHE) and the Research Foundation - Flanders (FWO), and the Special Research Fund of the Ghent University (BOF) for the financial support (GOA 01G01008).

\section{REFERENCES}

[1] http://www.ugct.ugent.be

[2] Dierick, M., Van Loo, D., Masschaele, B., Boone, M. and Van Hoorebeke, L. A LabVIEW (R) based generic CT scanner control software platform. Journal of X-Ray Science and Technology, 2010, 18(4): 451-461.

[3] Vlassenbroeck J, Dierick M, Masschaele B, Cnudde V, Van Hoorebeke L, Jacobs P. Software tools for quantification of X-ray microtomography. Nuclear Instruments \& Methods in Physics Research, Section A: Accelerators, Spectrometers, Detectors, and Associated Equipment. 2007, 580(1):442-5.

[4] Brabant L, Vlassenbroeck J, De Witte Y, Cnudde V, Boone MN, Dewanckele J, et al. Threedimensional analysis of high-resolution X-ray computed tomography data with Morpho+. Microscopy and Microanalysis. 2011, 17(2):252-63.

[5] Masschaele, B., Dierick, M., Van Loo, D., Boone, M.N., Brabant, L., Pauwels, E., Cnudde, V., Van Hoorebeke, L. HECTOR: A 240kV micro-CT setup optimized for research. Journal of Physics Conference series, proceedings of the 11th International Conference on X-Ray Microscopy, 2010, august 5-10, Shanghai, China. 